\title{
XXème CONGRES DE LA SALF (Société d'Andrologie de Langue Française)
}

11, 12 et 13 DECEMBRE 2003

CENTRE De CONFÉREnCes ORLEANS - France

ORGANISATION Dominique DELAVIERRE, Laurent CORCIA, Abdelhak MEDALLEL, Hussein IBRAHIM Michel PENEAU

\section{PROGRAMME PRELIMINAIRE}

Troubles de l'éjaculation

Andrologie de l'homme vieillissant Déficit androgènique lié à l'âge

Fertilité de l'homme vieillissant

Prostatites chroniques et syndrome douloureux pelvien chronique de l'homme

Contraception masculine

Infertilité sécrétoire et ICSI

Pathologie du veru montanum et du carrefour vésiculo-déférentiel

Biopsies testiculaires : registre français ?

Présentation orale des posters sélectionnés, des mémoires de DEA et DESS primés

Présentation des lauréats des bourses de recherche attribuées par la SALF

Symposiums satellites en partenariat avec l'industrie pharmaceutique

Soirée de GALA du 20ème Anniversaire de la SALF

RENSEIGNEMENTS

SECRETARIAT SCIENTIFIQUE

Dr Dominique DELAVIERRE Service d'UROLOGIE-ANDROLOGIE

CHR La Source BP 670945067 ORLEANS Cedex 2 (France)

Téléphone $33(0) 238514626$ Télécopie $33(0) 238514159$ Mail delav.uro@wanadoo.fr

\section{SECRETARIAT ADMINISTRATIF}

\section{ORLEANS CONGRES / SALF 2003}

1 rue du Président R. SCHUMAN 45074 ORLEANS Cedex (France)

Téléphone $33(0) 238569730$ Télécopie 33 (0)2 38569735 Mail OrleansCongres@orleansgestion.fr 


\section{CONGRÈS}

26-29APRIL 2003

2003 Testis Workshop : 'Functional Genomics of Male Reproduction'

Phoenix, Arizona, USA

Information : American Society of Andrology, 74 New Montgomery, Suite 230,

San Francisco, CA 94105, USA

29 APRIL-4 MARCH 2003

28th Annual Meeting of the American Society of Andrology

Phoenix, Arizona, USA

Information : American Society of Andrology, 74 New Montgomery, Suite 230,

San Francisco, CA 94105, USA

Tel: 415-764-4823, Fax: 925-472-5901, E-mail: asa@hp-assoc.com

\section{$2^{\circ}$ Forum Méditerranéen d'Andrologie}

\section{2-14 JUIN 2003}

Collioure-France

Renseignements : Dr Michel Schouman, 24 rue Pierret, Neuilly, France email :michel@schouman.com

JUILLET 2003

Annual Meeting of the Society for the Study of Fertility

Aberdeen, UK

Society@convention.co.jp

URL : http://www2.convention.co.jp/ichs2002

\section{9-22 JUILLET 2004}

International Congress of Endocrinology

Lisbon, Portugal

Maguelone G Forest,

DRI-INSERM-U.329

Hopital Debrousse, 29 rue Sur Bouvier, 69322 Lyon Cedex 05 France

Tel +33-4-7238-5848 ; Fax +33-4-7825-6168 ; Email forest@lyon151.inserm/fr

28-30 AUguSt 2003

Nordic Association for Andrology (NAFA) Annual Meeting 2003

Oslo, Norway

Information : http://www.ki.se/org/nafa/

\section{5-27 SEPTEMBER 2003}

$3^{\text {rd }}$ International Symposium on Testosterone : Action, Deficiency, Substitution Castle Elmau, Bavaria, Germany.

Information :Prof Dr med E Nieschlag FRCP ; email :nieschl@uni-muenster.de

2ND-4TH OCTOBER 2003

EEA International Symposium : Genetics of Male Infertility

Florence, Italy

Contact :c.krausz@dfc.unifi.it ;www.dfc.unifi.it/genetics-eea/ 